\title{
The Possibility of the Reseach on Yan-Zhao Culture from the Fang Yu Words in the Shuo Wen jiezi
}

\author{
Jing-xian MA and Shan-shan YAN \\ Hebei engineering university college of arts, the city of Handan 056038, China \\ Email:majingxian532@163.com
}

Keywords: Yan-zhao, Culture transformation, Fang Yu words in Shuo Wen jiezi.

\begin{abstract}
Yan-zhao culture formation mechanism, the development course, the regional differentiation and the refracted by the inherent regularity, in the development of the traditional Chinese culture will help explore conforms to the regional characteristics of the protection of traditional culture and development mechanism, to further promote and develop traditional culture. From language to talk about culture, itself has the necessity. This article tries from Fang Yu words in Shuo wen jiezi to discuss the possibility of Yan-zhao culture from the perspective of the research.
\end{abstract}

\section{Introduction}

Regional culture is a very old topic, also is a cross subject in modern science. Strictly speaking, to conscientiously study of zhao regional culture, the rise time is in the 1980s. After a period of academic accumulation, in the $1990 \mathrm{~s}$, Yao-Zhao culture field expands unceasingly, research results appear constantly, showing a preliminary boom. Researches on Yao-Zhao culture combine linguistics and profound national culture provides rich soil. Yao-Zhao culture and alien culture exchanges and integration is also reflected in Yao-Zhao region even the dialect words more than even Shuo wen jiezi. This paper aimed to explore Yao-Zhao culture from the language in the absorption of foreign culture, and with the alien culture and central plains wen-hua zhao exchange collision to verify the ductility of Yao-Zhao culture.

\section{Fang Yu Words in Shuo wen jiezi}

Fang $\mathrm{Yu}$ words refer to geographical scope to a limited amount of vocabulary, they include Chinese vocabulary and others. Belongs to Chinese vocabulary, Fang $\mathrm{Yu}$ words should be for the region's specific geographical areas.

Some Fang Yu words in Shuo Wen jiezi are Explicit and clear. Mr Ma Zonghuo published in the " $50 \mathrm{~s}$ "Shuo Wen words of dialect", he is the most good. The textual research of Mr Ma Zonghuo is that there are 174 dialect words in Shuo Wen jiezi. This article by $\mathrm{Ma}$ is on the basis of the continued researches, the controversial words out and missed joined, take-home contains Fang Yu word research according to is for 167.

However, according to the word Numbers is not equal to the number of dialect words. Because there are some dialect words for the same mean. For example "Qi" called “芋” as“莒”is put behind the word “莒”.Contain but not limited to, for example, Chu called it “唳”, Jin called it “䕱”, Qi called it “茞” is behind the word “圙”. There are some uncontained, for example, Chu called it “芰”, Qi called it “薢若”is behind the word“蔆”. So the number of the first word is167, but a total of 194 dialect words. Discussed in this paper, the "167" contained in article 194 dialect words, have belong 
to the dominant dialect words, namely, the writer Xu Shen clearly in the use of their area, recessive Fang $\mathrm{Yu}$ words do not discussed in this paper.

\section{Yanzhao Region Language in Shuo Wenjiezi Has the Possibility of Retained The Geographical Location of Its Own}

The spring and autumn period and the warring states period, hebei Yan and zhao is the main area. Yan (located in the northern hebei, its core region is xiadu (now hebei yihsien) area; Zhao is in the south of hebei, the core area of handan, the dividing line between the two countries for the baoding today. Yan culture and Zhao culture, together constitute the Yan-Zhao culture.

Yan-Zhao culture has the strong contemporary. It is not intermittent, generally referring to Hebei province from the ancient to now and including the Pre-Qin period of culture in the region. They linked to a certain geographical and cultural similarities.

\section{Fang Yu Words from Yanzhao Region in Shuo Wen jiezi}

Shuo Wenjiezi main has explanation of text, but also the sound, the meanings. $\mathrm{Xu}$ Shen adopted many rich informations in his book. So it is fair to say that Shuo Wen jiezi is a great linguistic book. Particularly, $\mathrm{Xu}$ shen has realized "the words are different sound, text different form". So the words in Shuo Wenjiezi included specific geographical areas is conscious by $\mathrm{Xu}$ Shen. The referenced words in specific geographical areas in Shuo Wenjiezi should be seen as Xu Shen achievements on dialect proverb.

Fang Yu words in the language of yanzhao recorded in Shuo Wenjiezi. The label which contains only one country name in Central China is "Yan", which contains several as "Zhao-Wei" "Jin-Zhao" "Yan-qi", which contains location name as "north".

\section{Fang Yu Words in Other Areas in Shuo Wen jiezi Assimilation of Yan-Zhao Region}

The origins of Fang Yu words in Shuo Wenjiezi can be traced back to, the Yan-Zhao culture collision with other regional culture can be finded. The collision of some dialect words might be cultural, which we can see the influence what maked Yan-Zhao culture changed on language and vocabulary.

It involves many geographical areas. For example, Chu, Qin, Sichuan, etc, and the number of minority languages such as the Qiang language, Vietnamese, Taiwanese and others. It can be drawing that in the pre-Qin period Chinese and non-Chinese language were closely related.

\section{Other Complications}

Some Fang Yu words in Shuo Wenjiezi are dialect words, Yan-Zhao culture connotation can be seen from the dialect words; Part of loanwords, the loanword is generalized, words from foreign culture, is the han words very well, can also be the Other ethnic groups words.

\section{The Ductility of Yan-Zhao Culture and Language Contact Theory}

Naming method of Yan-Zhao culture not only with regional more historic, contact Chinese history more; And the modern breath of Yan-Zhao culture is thick, it has no time limit, generally refers to the culture of today's hebei province since ancient times, 
this area is not only contains the pre-qin period, zhao culture originated from the warring states period, and it has another source. After into the long river of Chinese history and culture, the Yan-Zhao culture and Qi-Lu culture, Wu-Yue culture, Chu culture, Qin culture, etc, together constitute the Chinese pre-qin period of society and even the social culture of qin and han dynasties, panoramic view, the formation and development of the unity of the Chinese nation culture plays an irreplaceable important role in the process.

Over the past 20 years, the research of the Chinese language contact becomes a hotspot, becomes the focus of Chinese dialectology concern of linguistics, sociolinguistics, and nation. The so-called language contact mainly involves three types:

1. The foreign language with native language, such as English and Chinese.

2. The standard Chinese (mandarin) with dialect contact, or dialects contact with local dialect.

3. The contact of spoken and written. Above such as history and oral contact at that time.

According to the theory of language contact in Fang Yu words' study proves that the ductility of Yan-Zhao culture, namely the Yan-Zhao culture and its cultural contact, the fusion, the Yan-Zhao culture inheritance and development of its own.

\section{Conclusion}

The rise and development of Yan-Zhao cultural studies was inseparable with the study of China's traditional culture in the 1980s and 1990s. In the 21st century, the academic circle pays more attention to the study about Yan-Zhao culture, and has published a number of very influential publications of research results.

Written language is the record of history and culture, the language material is well-preserved fossil records, Chinese ancestors left precious cultural and historical data to future generations. Shuo We jiezi is a master of linguistics, but the inside of the record is the culture of the language in that time. Ancient can also through these records can be passed today. According to the purpose of this paper is to demonstrate Fang $\mathrm{Yu}$ words to study the feasibility of the regional culture, the relationship between Fang Yu words and the Yan-Zhao culture, the development and change of words confirm Yan-Zhao culture development and so on. These can be done to explore.

\section{Acknowledgement}

This research was financially supported by Social science fund project in hebei province. Its name is "The change of Yan-Zhao culture seen from the Fang Yu words in Shuo Wen jiezi". The number is HB14WX022.

\section{References}

[1] (Han Dynasty) Shen Xu; (Song Dynasty) Xuan Xu calibrated. Shuo Wenjiezi beijing: Zhonghua Book Company, 2004. (in Chinese)

[2] Zong-huo Ma. Textual research on dialect words of Shuo Wen jiezi, Beijing: Science book Company, 1959. (in Chinese)

[3] Jing-xian Ma. the pre-Qin period language relation seen from the dialects in Shuo Wen jiezi [J], Lan Zhou University,2008. (in Chinese) 
[4] Gong-jin Zhang. Cultural attributes of charactesr [J] Ethnic Chinese (1991). 